\title{
Lesión del nervio cubital tras fractura cerrada de antebrazo - dos casos clínicos
}

\section{Ulnar Nerve Injury after Closed Forearm Fracture - Two Cases Report}

\author{
Ricardo Kaempf de Oliveira ${ }^{1}$ Rafael Pegas Praetzel ${ }^{2,3}$ Leohnard Roger Bayer ${ }^{2,3}$ \\ Paulo Henrique Ruschel ${ }^{3,4}$ Augusto Heinen ${ }^{5}$ Pedro J. Delgado 6,7
}

${ }^{1}$ Grupo de Cirugía de Mano, Hospital da Criança Santo Antônio, Santa Casa de Misericórdia de Porto Alegre, Porto Alegre, RS, Brasil

${ }^{2}$ Cirugía de Mano, Hospital Mãe de Deus de Porto Alegre, Porto Alegre, RS, Brasil

${ }^{3}$ Cirugía de Mano, Hospital Santa Casa de Misericórdia de Porto

Alegre, Porto Alegre, RS, Brasil

${ }^{4}$ Hospital Moinhos de Vento, Porto Alegre, RS, Brasil

${ }^{5}$ Residencia en Ortopedia, Hospital Santa Casa de Misericórdia de

Porto Alegre, Porto Alegre, RS, Brasil

6 Cirugía de Mano y Miembro Superior, Hospital Universitário Madrid

Montepríncipe, Madri, España

${ }^{7}$ Universidad CEU San Pablo, Boadilla del Monte, Madri, España
Address for correspondence Ricardo Kaempf de Oliveira, MD, Rua Leopoldo Bier, 825, Conjunto 301, Porto Alegre, RS, CEP: 90620-100, Brasil (e-mail: ricardokaempf@gmail.com).

Rev Iberam Cir Mano 2017;45:120-124.

\section{Resumen}

\section{Palabras Clave}

- nervio ulnar

- lesiones nervio periférico

- lesiones del antebrazo

- fracturas de la ulna

\section{Abstract}

Las lesiones nerviosas asociadas a la fractura cerrada de los huesos del antebrazo son poco frecuentes y generalmente consisten en lesiones parciales del nervio mediano. Hay pocos casos publicados de una lesión grave del nervio cubital asociada a una fractura cerrada del antebrazo. Presentamos dos casos clínicos de lesiones del nervio cubital consecuencia de una fractura cerrada de antebrazo. Un diagnóstico precoz y la detección de los signos clínicos de gravedad son fundamentales, ya que influyen directamente en la actitud a tomar y en el resultado del tratamiento. Los casos que se presentan, se trataron de forma quirúrgica y se encontraron lesiones nerviosas severas. Es difícil estandarizar el tratamiento, ya que existen distintas causas de lesión nerviosa y un rango amplio de gravedad. Tras analizar la mayoría de los casos publicados, la recomendación inicial es esperar la mejora espontánea de cuatro a seis meses. Si después de ese período, no hay mejoría de la sintomatología neurológica, está indicado realizar una exploración microquirúrgica del nervio. Se revisan algunos signos de gravedad que pueden indicar un tratamiento quirúrgico precoz.

Nerve lesions associated to closed forearm bone fractures are rare, and usually consist of a partial injury to the median nerve. There are very few reports of a severe ulnar nerve injury associated to closed forearm fracture. We describe two cases of ulnar nerve lesion caused by closed forearm fracture. Early diagnosis and the importance of detecting red-flag clinical signs are stressed, as they directly influence the measures received

February 3, 2017

accepted

July 31,2017

published online

September 13, 2017
DOI https://doi.org/

10.1055/s-0037-1606284. ISSN 1698-8396.
Copyright (C 2017 Thieme Revinter

Publicações Ltda, Rio de Janeiro, Brazil
License terms

(c) (i) $\ominus$ (\$) 


\section{Keywords}

- ulnar nerve

- peripheral nerve injuries

- forearm injuries

- ulna fractures to follow and treatment outcome. Our patients were surgically treated, and severe nerve injuries were observed. It is hard to standardize the treatment, due to different causes of nerve lesion and the wide range of severity. By analyzing most reported cases, the initial recommendation is to wait for spontaneous improvement during four to six months. If after this time lapse there is no neurological symptom improvement, there is an indication for nerve microsurgical exploration. We have also reviewed some red-flag signs that may warrant early surgical treatment.

\section{Introducción}

El antebrazo es la localización más común de fractura en los niños. Es muy infrecuente la asociación de esas fracturas con las lesiones nerviosas, aunque se pueden observar en grandes fracturas abiertas con pérdida de partes blandas y en lesiones por armas de fuego. En las fracturas cerradas ese tipo de complicación se vuelve aún más excepcional. ${ }^{1,2}$

A pesar de esa asociación inusual, se debe estar siempre atento a la posibilidad de su aparición. La lesión, puede ocurrir tanto en el momento de producirse la fractura, por lesión directa, como posteriormente, por su atrapamiento en el foco de fractura con el intento de reducción. Por lo tanto, es importante definir el momento exacto del comienzo de los síntomas y detectar si ha sido poco después del traumatismo o después de la reducción. En ese tipo de lesiones, es importante un diagnóstico y una detección de los síntomas de gravedad precoz ya que, según el tipo de lesión y sus características, se pueden adoptar diferentes conductas. ${ }^{3-5}$

Dentro de las lesiones nerviosas asociadas a fracturas cerradas de antebrazo, la lesión del nervio cubital es aún más infrecuente. ${ }^{1-11}$ En el presente trabajo se describen dos casos clínicos en pacientes menores con fractura cerrada de los huesos del antebrazo asociados a la lesión del nervio cubital.

\section{Casos Clínicos}

\section{Paciente 1}

Paciente de 12 años, tras caída con una bicicleta, tuvo una fractura cerrada del antebrazo izquierdo moderadamente desplazada. Fue atendida inicialmente en un servicio de urgencias, donde se le colocó un yeso braquio-palmar para alinear el miembro, pero sin realizar maniobras de reducción. El mismo día, la paciente comenzó con debilidad y falta de sensibilidad en el territorio inervado por el nervio cubital. Tanto la paciente como los familiares no supieron indicar si los síntomas aparecieron después del traumatismo o de la colocación del yeso. En ese momento, se decidió realizar un tratamiento conservador manteniendo la inmovilización durante seis semanas. La fractura se consolidó sin gran deformidad, pero los síntomas de parálisis cubital evolucionaron a peor. La paciente fue atendida en nuestro servicio a los cinco meses de la lesión. En el examen físico presentaba los signos clásicos de lesión del nervio cubital, con parestesias y deformidad en garra del cuarto y quinto dedos (-Fig. 1). Los test de Froment, Bouvier y Wartenberg eran positivos. El electromiograma (EMG) demostró una neurotmesis del nervio cubital localizada entre el codo y la muñeca, sin signos de reinervación. Se indicó un tratamiento

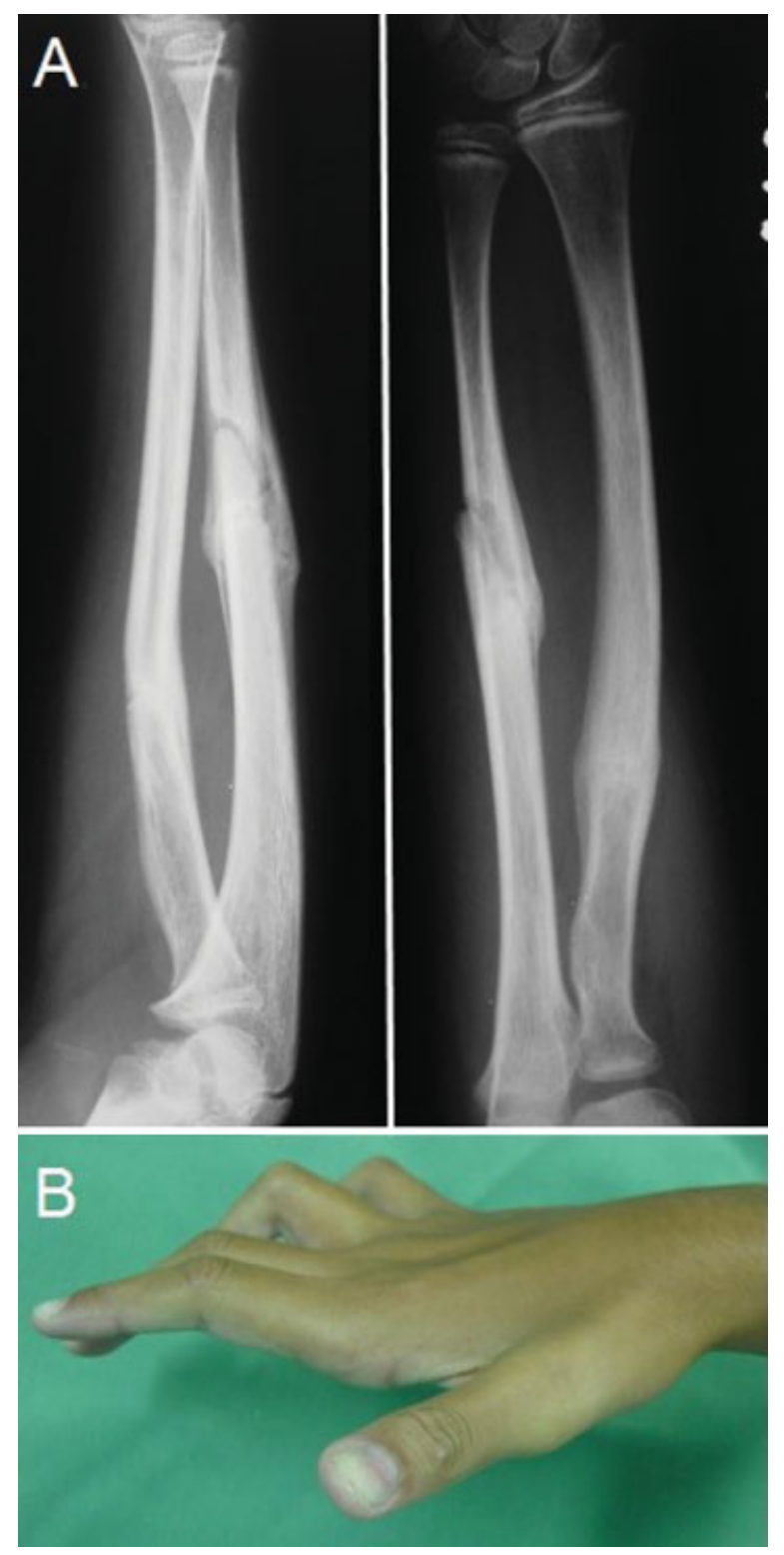

Fig. 1 Paciente de 12 años con fractura cerrada de antebrazo y lesión del nervio cubital. (A) Fractura consolidada sin gran deformidad. (B) En examen físico se observan los signos clínicos de lesión del nervio cubital, con la parestesia y la actitud en garra del cuarto y quinto dedos. 

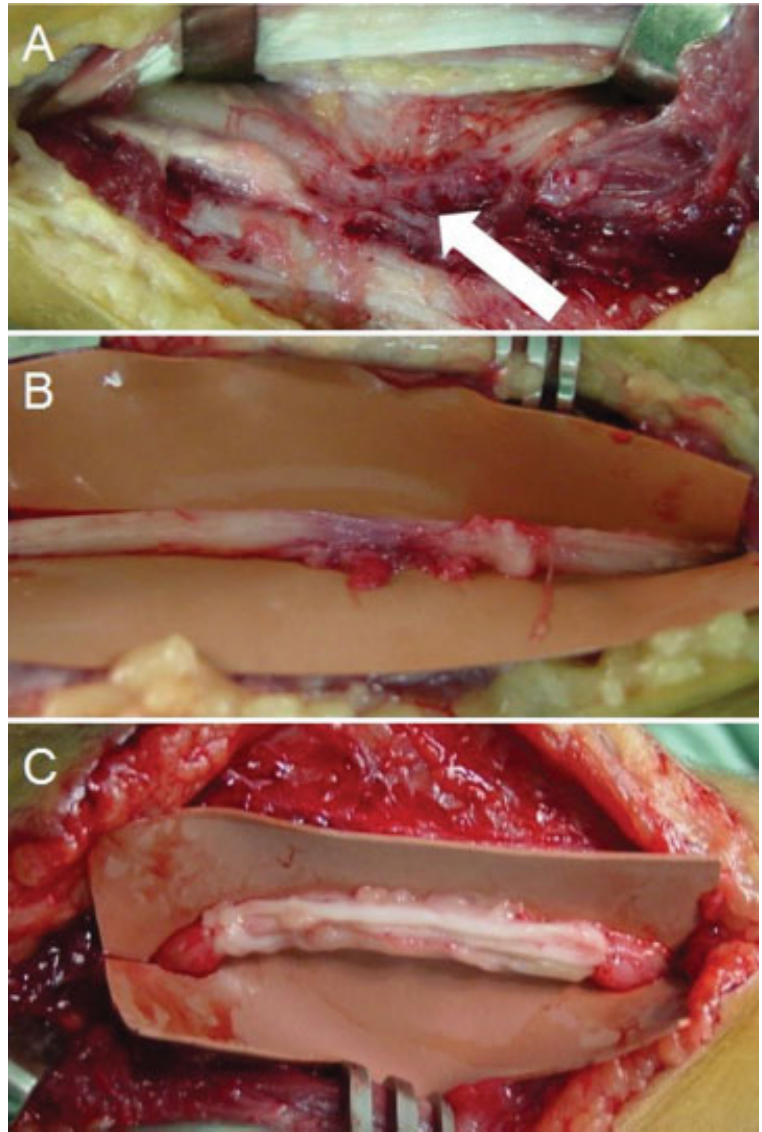

Fig. 2 Imágenes intraoperatorias caso 1. (A) Se observa la interposición del nervio en el interior del callo óseo y del foco de fractura (flecha blanca). (B) Lesión nerviosa completa sin viabilidad de ningún fascículo. (C) Reconstrucción con nervio sural suturado con el monofilamento de nylon 9/0 y cola de fibrina.

quirúrgico para la exploración del nervio. Durante el procedimiento se observó la interposición del nervio en el interior del callo y del foco de fractura, junto con la fascia del músculo flexor profundo de los dedos, sin existir ninguna lesión vascular. La disección microquirúrgica, con ayuda de gafas lupa, reveló una lesión completa del nervio, sin restos de fascículos viables en el área de la lesión. Tras la resección del tejido cicatricial y del neuroma, el defecto nervioso fue de tres centímetros. Se realizó la reconstrucción neural con injerto de nervio sural (cuatro fascículos), suturados con hilo monofilamento de nylon $9 / 0$ y cola de fibrina (-Fig. 2). La paciente evolucionó de forma favorable, con una corrección de la garra y mejora de la sensibilidad a los seis meses de la cirugía.

\section{Paciente 2}

Paciente de 17 años, acude al servicio de urgencias de nuestro hospital tras una caída con monopatín. Presentaba dolor y deformidad en el antebrazo, y limitación funcional del miembro superior. El examen neurovascular en el momento de la urgencia fue normal. Tras realizar el estudio radiográfico, se diagnosticó una fractura desplazada del antebrazo. Se realizó la reducción cerrada de la fractura bajo anestesia intrafocal y, una inmovilización con yeso (-Fig. 3). En el

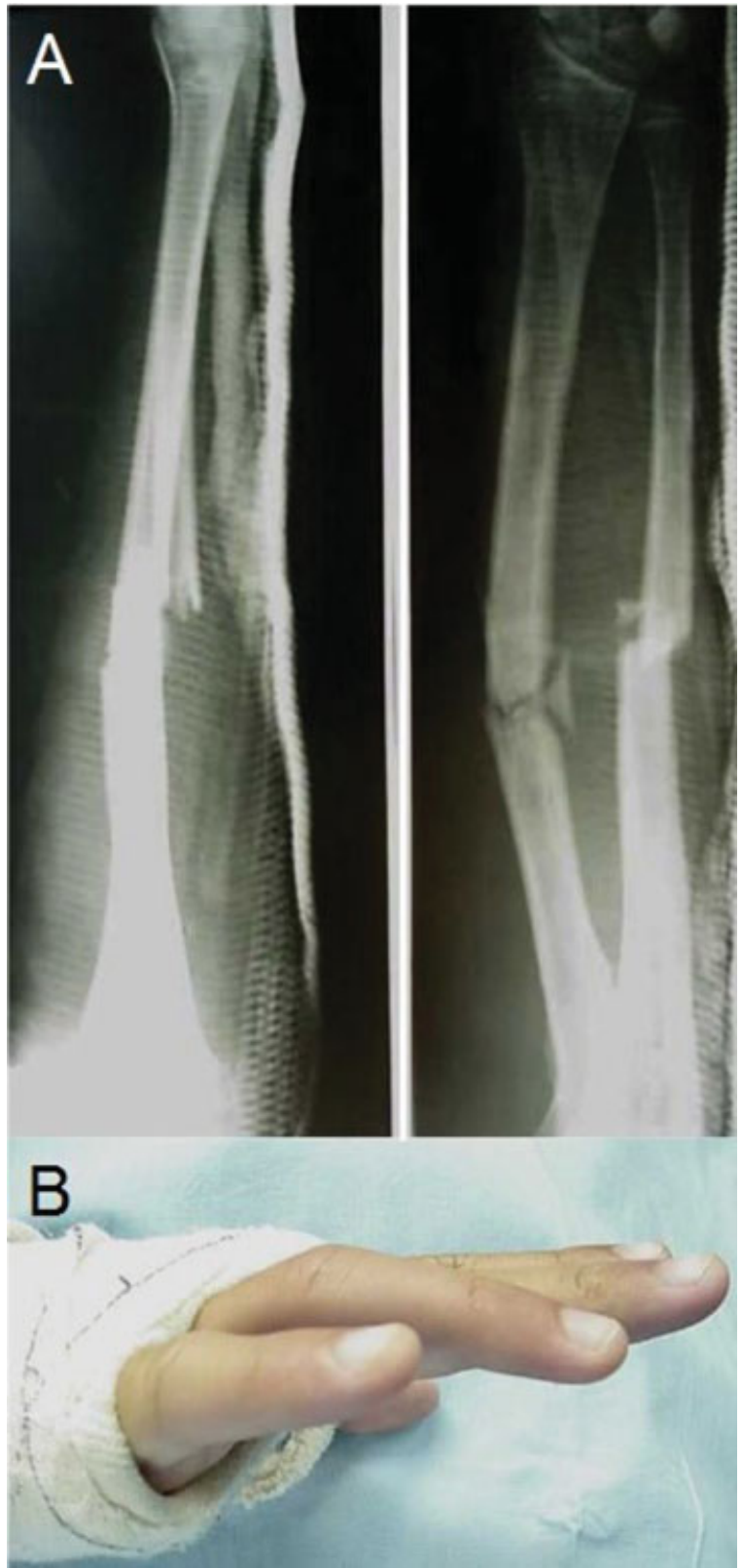

Fig. 3 (A) Imagen radiológica tras la reducción cerrada de la fractura. (B) Tras la reducción, el paciente comienza con clínica de lesión del nervio cubital.

control radiológico tras la reducción se comprobó una alineación correcta de la fractura, sin embargo, el paciente comenzó con debilidad en la mano, parestesias en el territorio del nervio cubital y deformidad en garra. Se sospechó de una lesión del nervio cubital, pero se decidió mantener una actitud expectante. Al no existir mejoría de la sintomatología después de tres semanas, se optó por la exploración quirúrgica. Se realizó una disección microquirúrgica del nervio cubital y se encontró incarcerado en el foco de la fractura. No se observó lesión de la arteria cubital o de la musculatura flexora. El nervio fue liberado del interior del foco de fractura, observándose una laceración parcial, con fascículos viables y lesión completa de otros. Se realizó una neurorrafia directa de los fascículos lesionados, con un hilo tipo monofilamento de nylon de 8/0. 


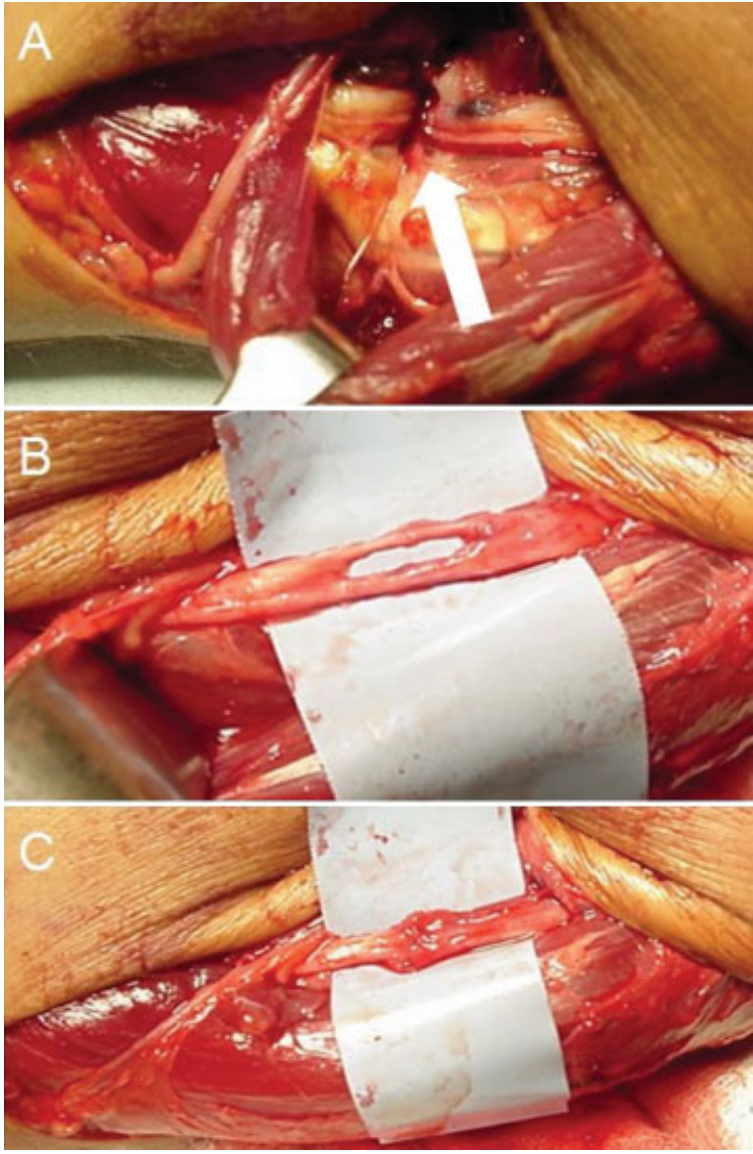

Fig. 4 Imágenes intraoperatorias caso 2. (A) El nervio se encuentra atrapado en el interior del foco de fractura (flecha blanca). (B) Se observa una continuidad de algunos fascículos y sección completa de otros. (C) Neurorrafia de los fascículos seccionados con monofilamento de $8 / 0$.

Durante el mismo acto quirúrgico, se realizó la osteosíntesis de la fractura del radio y del cúbito ( - Fig. 4). En el control clínico a los 12 meses, se observó una consolidación completa de la fractura y una movilidad normal del antebrazo, muñeca y mano. La garra cubital estaba corregida y normalizada los test de lesión del nervio cubital. El paciente todavía notaba una disminución de la sensibilidad del cuarto y quinto dedo, pero con prueba de discriminación de dos puntos de ocho milímetros y sin atrofia del pulpejo.

\section{Discusión}

Las fracturas de los huesos del antebrazo representan el 50\% de las fracturas pediátricas y pueden venir acompañadas de complicaciones bien conocidas, como la consolidación viciosa o el síndrome compartimental. ${ }^{1}$ Las lesiones nerviosas son inusuales y raramente se observan en niños en una fractura cerrada. Cuando ocurre, lo más común es que sean lesiones parciales y del nervio mediano. La lesión del nervio cubital asociada a fracturas del antebrazo pediátrico es excepcional. Aparte de los dos aquí presentados, sólo hemos encontrado en la literatura otros $12 \operatorname{casos}^{9}$ ( - Tabela 1 ). Otros casos descritos como fractura del antebrazo, en realidad presentaban una fractura de muñeca. ${ }^{2}$

Esa asociación excepcional, se debe a aspectos anatómicos del nervio cubital en el antebrazo y muñeca. En el antebrazo, el nervio discurre paralelo al músculo flexor profundo de los dedos y profundo al músculo flexor ulnar del carpo. Es decir, esos músculos suponen una protección mecánica del nervio. Además, Clarke y Spencer ${ }^{12}$ han comprobado que el nervio cubital posee una razonable movilidad en el antebrazo y en el canal de Guyon, mucho mayor que la del nervio mediano en el interior del túnel carpiano. Ese hecho permite al nervio desplazarse y evitar una posible lesión causada por los fragmentos óseos. Stahl y col. ${ }^{5}$ demostraron otro factor que influye en esa baja incidencia en niños y es que la mayoría de las veces son traumatismos de baja energía, permitiendo que el nervio "escape" de los fragmentos óseos en el momento del accidente. Sin embargo, por la gran proximidad del nervio cubital al cúbito en los tercios medio y distal del antebrazo,

Tabela 1 Resumen de artículos en los que se han publicado lesiones del nervio cubital asociadas a una fractura de antebrazo

\begin{tabular}{|c|c|c|c|c|}
\hline Autor & Edad & Tratamiento inicial & $\begin{array}{l}\text { Tiempo hasta } \\
\text { la cirugía }\end{array}$ & Tipo de Lesión \\
\hline Prosser y Hooper 6 & 13 & Fijación interna & 10 & Atrapamiento en el foco \\
\hline \multirow[t]{3}{*}{ Stahl y col. ${ }^{5}$} & 10 & Reducción cerrada & 10 & Compresíon por fibrosis \\
\hline & 15 & Reducción cerrada & 16 & Compresíon por fibrosis \\
\hline & 9 & Fijación interna y reparación del nervio & 0 & Lesión parcial \\
\hline Hirasawa y col. $^{3}$ & 13 & Reducción cerrada & 13 & Atrapamiento en el foco \\
\hline Torpey y col. ${ }^{8}$ & 15 & Fijación interna y reparación del nervio & 0 & Lesión parcial \\
\hline \multirow[t]{2}{*}{ Neiman y col. ${ }^{7}$} & 12 & Reducción cerrada & No operado & Lesión parcial \\
\hline & 11 & Reducción cerrada & No operado & Lesión parcial \\
\hline Saganuma y col. ${ }^{4}$ & 12 & Fijación interna & 9 & Lesión parcial \\
\hline $\mathrm{Pai}^{10}$ & 13 & Reducción cerrada & 12 & Atrapamiento en el foco \\
\hline Pasque y col. ${ }^{11}$ & 19 & Inmobilización & No operado & Lesión parcial \\
\hline Schwartsmann y col. ${ }^{9}$ & 17 & Reducción cerrada & 3 & Atrapamiento en el foco \\
\hline
\end{tabular}

Edad en años, tiempo hasta la cirugía en semanas. 
todos los factores de protección pueden no ser suficientes. En el intento de reducción de la fractura, cuando se realiza la maniobra que aumenta la deformidad, la angulación ósea puede causar un daño directo al nervio o su incarceración en el interior del foco de la fractura.

Hirasawa y col. ${ }^{3}$ sugieren diferentes mecanismos que pueden causar la lesión. Los pacientes que presentan el déficit nervioso justo después de la fractura, tienen una alta posibilidad de que la lesión sea parcial y haya sido causada por el trauma directo de una espícula ósea. Cuando la aparición de la lesión es tras la reducción de la fractura, la mayoría de los pacientes presentan un atrapamiento del nervio en el foco. Por último, existen lesiones tardías, generalmente causadas por una compresión progresiva del nervio en el tejido cicatricial y callo óseo. ${ }^{5}$

Algunas características de la fractura pueden aumentar la sospecha de lesión. La primera es la angulación anterior. ${ }^{3}$ La otra es la ausencia de formación del callo óseo, conocido como signo de Matev. ${ }^{13}$ Éste se describió originalmente por la interposición del nervio mediano en una fractura-luxación de codo y ocurre por la interposición del nervio en el foco de fractura.

Otras pruebas son de poca ayuda en la toma de decisiones de esas lesiones. El EMG en fase aguda es incapaz de diferenciar si la lesión del nervio es parcial o total. Con el uso de la resonancia magnética (RM), Amit y col. ${ }^{2}$ han descrito la posibilidad de detectar si hay o no atrapamiento del nervio en el foco de la fractura. Sin embargo, en nuestro medio, hemos encontrado dificultad para obtener esta claridad de imagen y definición. El uso de ultrasonografía de alta resolución también ha sido publicado como útil en la evaluación de la gravedad de lesiones traumáticas de nervio periférico, pero todavía están en fase experimental. ${ }^{11}$

Fernandez y Segal ${ }^{1}$ explican que además de la lesión nerviosa, las fracturas del tercio medio del cúbito pueden causar la lesión de la musculatura del flexor profundo de los dedos. Esa lesión y su contractura por la cicatrización causan una incapacidad para extender los dedos, principalmente el $4^{\circ}$ y $5^{\circ}$, pudiendo ser confundido con la actitud en garra consecuencia de la lesión del nervio ulnar o con la contractura causada como secuela de síndrome compartimental y Volkmann.

En base a la gravedad de la lesión nerviosa, el tratamiento varía desde una actitud conservadora, hasta la exploración quirúrgica del nervio, con posible neurolisis o reparación microquirúrgica. ${ }^{2-4,6,7}$ Por ello es de extrema importancia, en la primera atención, realizar un examen físico completo, con especial atención a la exploración nerviosa. Es importante remarcar que en niños el dolor y la deformidad de la fractura pueden hacer difícil la exploración neurológica. ${ }^{3}$ Una lesión aguda inmediatamente posterior a la fractura, sugiere una lesión parcial con buen pronóstico y la mayoría de las veces mejora espontáneamente sin precisar cirugía. Por el contrario, la aparición de clínica después de la reducción sugiere el incarceramiento del nervio en el foco, por lo que está indicado un procedimiento quirúrgico precoz. ${ }^{2-4,10}$

Stahl y col. ${ }^{5}$ indican el tratamiento quirúrgico precoz y la exploración del nervio en los casos de fractura irreductible, lesión asociada de la arteria cubital y fractura abierta. Se justifica la cirugía precoz ya que proporciona la posibilidad de realizar una reparación directa de la lesión, la extracción de fragmentos óseos que estén lesionando directamente el nervio o la retirada del nervio del interior del foco de la fractura. Explican que, en esos casos, realizar un tratamiento expectante puede hacer necesario el uso posterior de injertos nerviosos, que hace que la recuperación completa sea mucho más difícil. Hirasawa y col. ${ }^{3}$ revisaron el tema y sugieren algunos signos y características clínicas de gravedad del nervio cubital con interposición en el interior del foco de la fractura. Esas características son: alteración motora y sensitiva justo después de la maniobra de reducción, empeoramiento progresivo del déficit neurológico y deformidad angular anterior de la fractura, con ausencia de formación de callo óseo en el foco (signo de Matev), estando en esos casos, indicado el tratamiento quirúrgico.

Al existir diferentes causas de lesión nerviosa y una gran variación en la gravedad, es difícil uniformizar el tratamiento. Al analizar la gran mayoría de los casos publicados, nuestra recomendación es esperar la mejoría espontánea durante cuatro a seis meses. Si después de ese período no hay mejoría, se indicaría la exploración microquirúrgica del nervio.

\section{Conflicto de Intereses}

Los autores declaran que no hay ningún conflicto de intereses.

\section{Bibliografía}

1 Fernandez ML, Segal LS. Entrapment of the flexor digitorum profundus following paediatric forearm fractures. Acta Orthop Belg 2007;73(06):780-784

2 Amit B, Ashish D, Vinit V, et al. Missed ulnar nerve injury and closed forearm fracture in a child. Chin J Traumatol 2013;16(04):246-248

3 Hirasawa H, Sakai A, Toba N, Kamiuttanai M, Nakamura T, Tanaka K. Bony entrapment of ulnar nerve after closed forearm fracture: a case report. J Orthop Surg (Hong Kong) 2004;12(01):122-125

4 Suganuma S, Tada K, Hayashi H, Segawa T, Tsuchiya H. Ulnar nerve palsy associated with closed midshaft forearm fractures. Orthopedics 2012;35(11):e1680-e1683

5 Stahl S, Rozen N, Michaelson M. Ulnar nerve injury following midshaft forearm fractures in children. J Hand Surg [Br] 1997;22 (06):788-789

6 Prosser AJ, Hooper G. Entrapment of the ulnar nerve in a greenstick fracture of the ulna. J Hand Surg [Br] 1986;11(02):211-212

7 Neiman R, Maiocco B, Deeney VF. Ulnar nerve injury after closed forearm fractures in children. J Pediatr Orthop 1998;18(05):683-685

8 Torpey BM, Pess GM, Kircher MT, Faierman E, Absatz MG. Ulnar nerve laceration in a closed both bone forearm fracture. J Orthop Trauma 1996;10(02):131-134

9 Schwartsmann CR, Ruschel PH, Huyer RG. Paralisia do nervo ulnar seguida de fratura dos ossos do antebraço. Rev Bras Ortop 2016;51:475-477

10 Pai VS. Injury of the ulnar nerve associated with fracture of the ulna: A case report. J Orthop Surg (Hong Kong) 1999;7:73-75

11 Pasque CB, Pearson C, Margo B, Ethel R. Distal ulna fracture with delayed ulnar nerve palsy in a baseball player. Am J Orthop 2016;45(02):86-88

12 Clarke AC, Spencer RF. Ulnar nerve palsy following fractures of the distal radius: clinical and anatomical studies. J Hand Surg [Br] 1991;16(04):438-440

13 Matev I. A radiological sign of entrapment of the median nerve in the elbow joint after posterior dislocation. A report of two cases. J Bone Joint Surg Br 1976;58(03):353-355 